\title{
Exercise capacity in asymptomatic patients with significant primary mitral regurgitation: independent effect of global longitudinal left ventricular strain
}

\author{
Amgad Mentias, Alaa Alashi, Peyman Naji, A. Marc Gillinov, L. Leonardo Rodriguez, Tomislav \\ Mihaljevic, Rakesh M. Suri, Richard A. Grimm, Lars G. Svensson, Brian P. Griffin, Milind Y. Desai \\ Heart Valve Center, Heart and Vascular Institute, Cleveland Clinic, Cleveland, OH, USA \\ Contributions: (I) Conception and design: A Mentias, AM Gillinov, LL Rodriguez, T Mihaljevic, RM Suri, BP Griffin, MY Desai; (II) Administrative \\ support: M Desai; (III) Provision of study materials or patients: AM Gillinov, LL Rodriguez, T Mihaljevic, RM Suri, RA Grimm, LG Svensson, BP \\ Griffin, MY Desai; ;(IV) Collection and assembly of data: A Mentias, A Alashi, P Naji, MY Desai; (V) Data analysis and interpretation: All authors; \\ (VI) Manuscript writing: All authors; (VII) Final approval of manuscript: All authors. \\ Correspondence to: Milind Y. Desai, MD. Heart and Vascular Institute, Department of Cardiovascular Medicine, Cleveland Clinic, 9500 Euclid \\ Avenue, Desk J1-5, Cleveland, OH 44195, USA. Email: desaim2@ccf.org.
}

Background: Despite preserved left ventricular ejection fraction (LVEF), patients with significant primary mitral regurgitation (MR) often have reduced exercise capacity. In asymptomatic patients with $\geq 3+$ primary MR undergoing rest-stress echocardiography (RSE), we sought to evaluate the incremental impact of left ventricular global longitudinal strain (LV-GLS) on exercise capacity.

Methods: A total of 660 asymptomatic patients with $\geq 3+$ primary MR, non-dilated LV and LVEF $\geq 60 \%$ (mean age, $57 \pm 14$ years, $66 \%$ men, body mass index or BMI $25 \pm 4 \mathrm{~kg} / \mathrm{m}^{2}$ ) who underwent RSE at our center between 2001 and 2013 were included. Standard RSE data were obtained. Average resting LV-GLS was measured using Velocity Vector Imaging.

Results: Mean mitral effective regurgitant orifice, resting right ventricular systolic pressure (RVSP) and LV-GLS were $0.45 \pm 0.2 \mathrm{~cm}^{2}, 31 \pm 12 \mathrm{mmHg}$ and $-21.7 \% \pm 2 \%$, respectively; $28 \%$ had flail mitral leaflet. Mean metabolic equivalents (METs) and post-stress RVSP were $9.9 \pm 3$, and $46 \pm 15 \mathrm{mmHg} ; 28 \%$ achieved $<100 \%$ age-gender predicted METs. No patient had ischemia or significant arrhythmias. On logistic regression, resting LV-GLS [odds ratio (OR), 1.40, 95\% confidence interval (CI): 1.21-1.55, BMI (OR, 1.11, 95\% CI: 1.06-1.17)] and resting RVSP 1.22 (1.02-1.49) were independent predictors of exercise capacity. Area under the curve for association between $100 \%$ age-gender predicted METs and various factors were as follows: (I) $\mathrm{BMI}$ (0.60, 95\% CI: 0.55-0.65, P<0.001); (II) resting RVSP (0.57, 95\% CI: 0.52-0.62, P=0.006) and LV-GLS (0.66, 95\% CI: 0.61-0.70, $\mathrm{P}<0.001)$.

Conclusions: In asymptomatic patients with $\geq 3+$ primary MR, non-dilated LV and preserved LVEF, LVGLS is independently associated with exercise capacity, beyond known predictors.

Keywords: Mitral regurgitation (MR); exercise capacity; global LV longitudinal strain

Submitted Feb 17, 2018. Accepted for publication Apr 02, 2018.

doi: $10.21037 /$ cdt.2018.05.03

View this article at: http://dx.doi.org/10.21037/cdt.2018.05.03

\section{Introduction}

In patients with significant primary mitral regurgitation (MR), previous reports have demonstrated that exercise echocardiography aids in symptom evaluation, risk stratification, decision making (for delaying of surgery, including in those with mid-late systolic MR) (1-3). In addition, decreased functional capacity (FC) on stress testing (4), exercise-induced pulmonary hypertension (5) and MR 
severity (6) have been associated with worse outcomes. Based on available data, exercise echocardiography gets a class IIa recommendation for assessment of MR patients (7). Several smaller studies have reported different and conflicting predictors of FC (4,8-15). Some reports have suggested that age, gender, and exercise cardiac output, early diastolic mitral annulus velocity, preoperative sinus rhythm, LV diastolic dysfunction, atrial fibrillation, forward stroke volume, exercise right ventricular systolic pressure (RVSP), low left atrial (LA) strain, brain natriuretic peptide predict FC, while LV ejection fraction, LA size, LV dimensions, mitral effective regurgitant orifice area (EROA), mitral regurgitant fraction, rest and exercise RVSP did not predict FC.

Assessment of left ventricular global longitudinal strain (LV-GLS), using speckle tracking echocardiography provides another quantitative assessment of LV contractile function that may be more sensitive to early reduction in $\mathrm{LV}$ function than left ventricular ejection fraction (LVEF) (16). We have recently demonstrated that a combination of resting LV-GLS and FC provide incremental prognostic utility in asymptomatic patients with severe MR (17). We hypothesized that if resting LV-GLS is a marker of intrinsic LV dysfunction, this dysfunction may translate into impaired FC in such patients. Hence, in asymptomatic patients with severe $M R$, non-dilated $L V$ and preserved $L V$ ejection fraction, we sought to: (I) determine predictors of impaired FC; and (II) determine if resting LV-GLS is associated with impaired FC, which is independent of other established predictors.

\section{Methods}

\section{Study population}

This was an observational cohort study of 660 consecutive asymptomatic patients with $\geq 3+$ primary myxomatous MR, non-dilated LV (indexed LV end-diastolic dimension $<3.3 \mathrm{~cm} / \mathrm{m}^{2}$ and absolute $\mathrm{LV}$ end systolic dimension $<4 \mathrm{~cm}$ ) and preserved LVEF (LVEF $\geq 60 \%$ ), who were seen and evaluated at our tertiary care center between January 2000 and December 2011. All patients included in this study had a comprehensive resting and treadmill exercise echocardiography performed at the time of initial clinical evaluation (within 24 hours of each other). In the current study time-frame, there were 1,694 total patients with $\geq 3+M R$ who underwent treadmill exercise echocardiography. We excluded the following 887 patients: those with symptoms, obstructive coronary artery disease, any documented baseline atrial fibrillation, more than mild aortic valve disease or mitral stenosis, functional MR, rheumatic heart disease, hypertrophic obstructive cardiomyopathy or history of mitral valve surgery. No patient had resting bundle branch block or pacemaker. We also excluded patients in whom poor image quality precluded adequate strain assessment $(\mathrm{n}=147)$. The study was approved by the institutional review board. Baseline data were prospectively entered into the electronic medical record, at the time of the initial encounter, and manually extracted for the current study. Society of Thoracic Surgeons (STS) score was calculated in all patients. Ethical statement: The study was approved by the institutional review board with waiver of individual informed consent.

\section{Resting and exercise echocardiography}

All patients underwent comprehensive echocardiograms using commercial instruments (Philips Medical Systems, N.A., Bothell, Wash and Siemens Medical Solution USA Inc., Malvern, PA, USA). LV ejection fraction, indexed $\mathrm{LV}$ dimensions and left atrial area were measured at rest according to guidelines (18). Severity of MR was ascertained using multiple previously described techniques (19). This included qualitative visual assessment (as a \% of left atrial size) and measurement of EROA and vena contracta width. Because of severity of MR, diastolic function was not reported. However, we recorded E/e' ratio. Presence of flail mitral leaflet was recorded. Right ventricular systolic function was measured qualitatively (normal, mild, moderate or severe). RVSP was measured at rest, according to guidelines, using the following formula: $4 \times$ [tricuspid regurgitant velocity $(\mathrm{m} / \mathrm{sec})]^{2}+$ right atrial pressure $(\mathrm{mmHg})(20)$. Right atrial pressure was estimated from inferior vena cava (IVC) diameter and respiratory changes as follows: $3 \mathrm{mmHg}$ (IVC $\leq 2.1 \mathrm{~cm}$, collapse with sniff $>50 \%$ ), $8 \mathrm{mmHg}$ (IVC $>2.1 \mathrm{~cm}$, collapse $>50 \%$ ) and $15 \mathrm{mmHg}($ IVC $>2.1 \mathrm{~cm}$, collapse with sniff $<50 \%)(20)$.

Subsequently, patients underwent treadmill echocardiography using Bruce or modified Bruce protocols, as appropriate. The test was terminated due to symptoms and not at achievement of target heart rate. Blood pressure, heart rate and electrocardiographic measurements were made at rest, at 1 minute intervals and for $\geq 6$ minutes in recovery. Maximal predicted heart rate (220-age), \%-predicted maximal heart rate and number of metabolic equivalents (METS) achieved were recorded. 
We calculated expected METs, based on age and gender, as previously described (1), using the Veterans Affairs cohort formula [predicted METs $=18-(0.15 \times$ age $)$ ] and the St. James Take Heart Project formula [predicted METs $=14.7-(0.13 \times$ age $)]$, for men and women, respectively. We subsequently calculated the following ratio: (METS achieved/age-gender expected METS) $\times 100$. Immediately following exercise, peak-stress echocardiographic images were acquired, according to guidelines (21), and the following parameters were assessed: regional wall motion abnormalities for evaluation of ischemia and peak-stress RVSP. We also evaluated for changes in LV cavity size (increase, decrease or no change), suggestive of presence or absence of contractile reserve. Major (sustained ventricular or atrial arrhythmias associated with severe symptoms, hemodynamic compromise, or need for cardioversion) and minor events (decrease in blood pressure, transient symptoms, or nonsustained arrhythmias) were recorded.

\section{$L V-G L S$}

LV-GLS measurements were obtained from baseline resting transthoracic echocardiograms using gray-scale images recorded in apical 2, 3 and 4-chamber views. LV-GLS was analyzed offline using Velocity Vector Imaging (Syngo Velocity Vector Imaging, Siemens Medical Solutions, Mountain View, CA, USA), as previously described (22). All raw data was stored in DICOM format without compression. The frame rate was at least 30 frames/second. After manual definition of the LV endocardial border, the endocardium was automatically tracked throughout the cardiac cycle. LV-GLS was obtained by averaging all segmental strain values from all 3 apical views. Peak global strain was defined as the peak negative value on the strain curve during the entire cardiac cycle. All measurements were made off-line by an investigator blinded to all clinical and demographic information (AM). Measurements were performed and averaged over 3 cardiac cycles. As the reported LV-GLS values are negative, a lower absolute number represented a worse value than higher (Figure 1A).

\section{Statistical analysis}

Continuous variables are expressed as mean $\pm \mathrm{SD}$, or median and inter-quartiles for skewed distributions, and compared using the Student $t$-test or analysis of variance (for normally distributed variables) or the Mann-Whitney test (for non-normally distributed variables). Categorical data is expressed as percentage and compared using Chi Square test or Fisher's Exact test, as appropriate. Intra and interobserver variability for LV-GLS measurements was assessed using intraclass correlation coefficients (ICC). Association between continuous variables was tested using Spearman's correlation coefficient. Univariable and multivariable logistic regression analysis were performed to test the association between various potential factors and $100 \%$ agegender predicted METs. Only factors with a $\mathrm{P}$ value $<0.1$ on univariable analysis were considered for multivariable model; $100 \%$ of age-gender predicted METs was utilized as a cutoff for logistic regression analysis, as we have previously reported it to be a cutoff demonstrating incremental prognostic value for the hard endpoint of death (17). In addition, we performed receiver operator characteristic (ROC) curve analysis to test the association between 100\% age-gender predicted METs and various significant factors observed on logistic regression analysis (individual as well as incremental). For incremental association between 100\% age-gender predicted METs and various significant factors, we entered the sequential probabilities obtained on logistic regression as the "test variable" on ROC curve analysis. Area under the curve (AUC) was reported for ROC curve analysis [along with $95 \%$ confidence intervals (CI)]. Statistical analysis was performed using SPSS version 11.5 (IBM Corp). A P value $<0.05$ was considered significant.

\section{Results}

Baseline clinical characteristics for the study population are shown in Table 1. Mean STS score was very low at 1.5 \pm 1.1 . Resting and treadmill echocardiographic data are shown in Table 2. By study design, all patients were asymptomatic with $\geq 3+M R$, and had normal $L V$ dimensions and preserved LVEF. Within the population, a majority (468 or $64 \%$ ) of the patients had a dilated left atrium and a total of 249 (38\%) patients had resting RVSP $\geq 35 \mathrm{mmHg}$. Median LV-GLS in the study population was $-21.7 \%$ (interquartile range, $-20.63 \%$ to $-22.98 \%$, maximum value of $-27.6 \%$ and minimum of $-17.2 \%)$. The ICC for intra-observer (MD) and inter-observer (AM and MD) reproducibility of resting LV-GLS measurements were $0.89(0.81-0.96)$ and 0.86 (0.71-0.92), respectively (both $\mathrm{P}<0.001)$. There was a weak correlation between LV-GLS and LVEF ( $\mathrm{r}=-0.11, \mathrm{P}=0.03$ ).

Patients terminated the stress test due to generalized fatigue. There were no significant arrhythmias, syncope or deaths during exercise, and by study design (excluding patients with coronary artery disease), there were no 

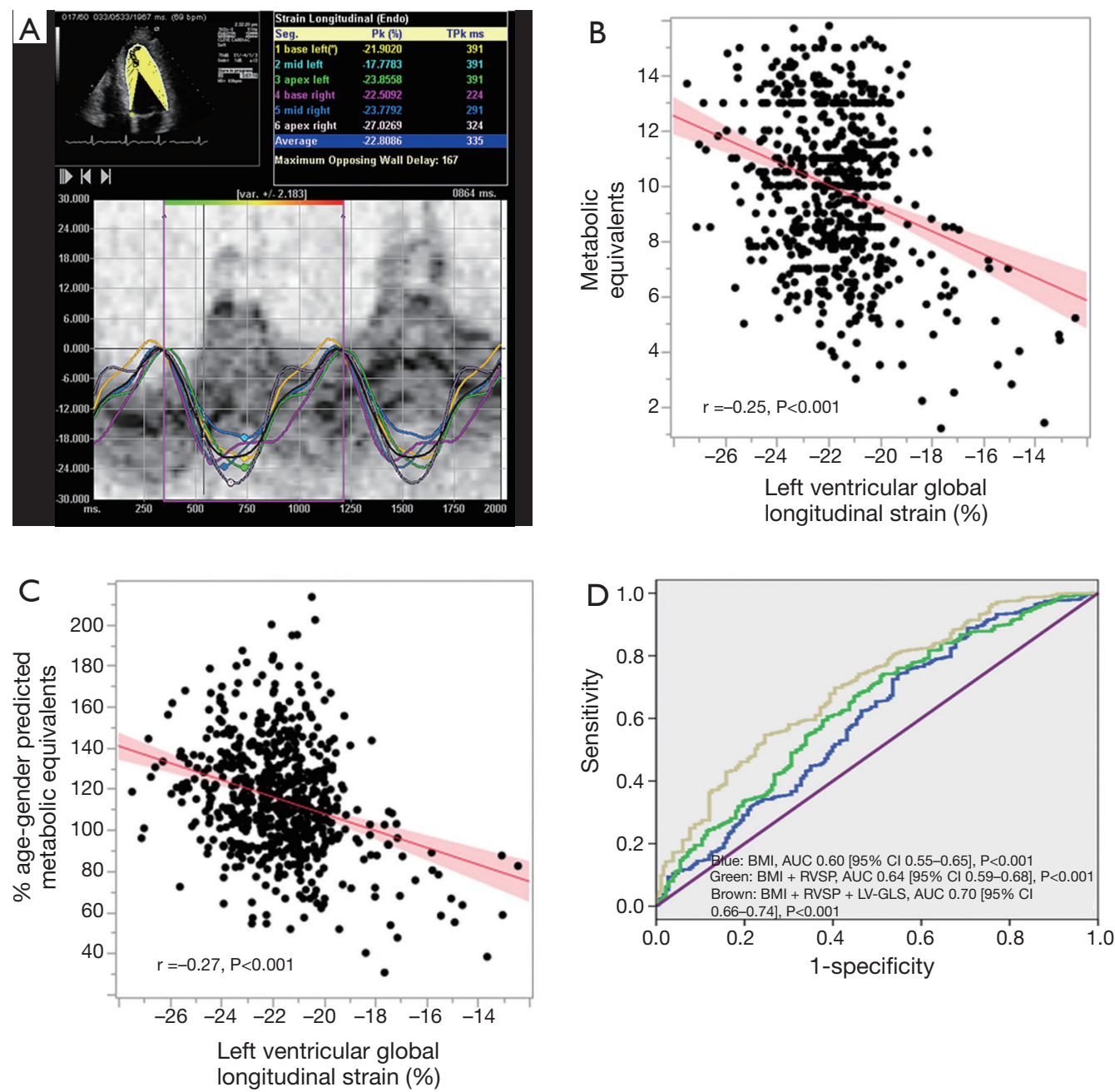

Figure 1 Data and images from the current study. (A) Representative example of left ventricular global longitudinal strain (LV-GLS) assessment in the study sample; (B) correlation between LV-GLS and metabolic equivalents (METS); (C) correlation between LV-GLS and $\%$ age gender predicted METS; (D) receiver operator characteristic curves in the study sample showing incremental value for association with achieving $100 \%$ age-gender predicted METS. BMI, body mass index; RVSP, right ventricular systolic pressure.

patients with an ischemic response to stress. Also, 110 (17\%) had post-stress RVSP $\geq 60 \mathrm{mmHg}$. There was a weak but significant correlation between LV-GLS and maximum METS (beta $=-0.25, \mathrm{P}<0.001$ ) or $\%$ age-gender predicted METs (beta $=-0.27, \mathrm{P}<0.001$, Figure $1 B, C$ ). Out of the 183 patients with $<100 \%$ predicted METs, majority of the patients ( $\mathrm{n}=114$ or $62 \%$ ) had LV-GLS worse than the median of $-21.7 \%$.

Univariable and multivariable logistic regression analyses were performed to determine the association between $100 \%$ age-gender predicted METs and various potential relevant predictors of FC. The data is shown in Table 3. On multivariable logistic regression analysis, higher body mass index (Wald statistic 17.6, $\mathrm{P}<0.001$ ), higher resting RVSP (Wald statistic 5.7, $\mathrm{P}=0.02$ ) and worse LV-GLS (Wald statistic 39.6, $\mathrm{P}<0.001)$ were independently associated with not achieving $100 \%$ age-gender predicted METs, while parameters like indexed LV dimensions, $L V$ ejection fraction, presence of flail leaflet, mitral regurgitant orifice area and indexed LA area had no significant association.

On ROC curve analysis, AUC's for association between $100 \%$ age-gender predicted METs and various individual relevant factors were as follows: (I) body mass index [0.60 (95\% CI: 0.55-0.65), $\mathrm{P}<0.001]$; (II) resting RVSP [0.57 (95\% CI: 0.52-0.62), P=0.006] and LV-GLS [0.66 (95\% CI: $0.61-0.70), \mathrm{P}<0.001]$. To test whether 
Table 1 Baseline characteristics of study sample $(n=660)$

\begin{tabular}{lc}
\hline Variable & Values \\
\hline Age (years) & $57 \pm 14$ \\
Male gender & $432(65 \%)$ \\
Body mass index $\left(\mathrm{kg} / \mathrm{m}^{2}\right)$ & $25 \pm 4$ \\
Hypertension & $296(45 \%)$ \\
Diabetes mellitus & $22(3 \%)$ \\
Hyperlipidemia & $262(40 \%)$ \\
Stroke & $17(3 \%)$ \\
Smoker & $264(40 \%)$ \\
ACE-I or ARBs & $230(35 \%)$ \\
Beta blockers & $188(28 \%)$ \\
Calcium channel blockers & $48(7 \%)$ \\
STS score & $1.5 \pm 1.1$ \\
Serum creatinine (mg/d) & $1.1 \pm 0.4$ \\
\hline ACE-l, angiotensin converting enzyme
\end{tabular}

ACE-I, angiotensin converting enzyme inhibitor; ARB, angiotensin receptor blocker; STS, Society of Thoracic Surgeons.

the association between LV-GLS and 100\% age-gender predicted METs was independent of other relevant factors (body mass index and resting RVSP), we performed additional ROC curve analysis utilizing the incremental probabilities obtained on logistic regression analysis. The ROC curves are shown in Figure 1D. LV-GLS significantly increased the predictive association with $<100 \%$ age-gender predicted METs, over and above known factors like body mass index and resting RVSP.

\section{Discussion}

In the current study of asymptomatic patients with $\geq 3+$ primary $M R$, non-dilated $L V$ and preserved $L V$ ejection fraction, we demonstrate that $(28 \%)$ patients had a subnormal exercise capacity on treadmill stress testing despite considering themselves asymptomatic in their daily life. We also demonstrate that resting LV-GLS had a highly significant association with age-gender predicted FC in this study population, independent (and incremental) of other known factors like resting RVSP and body mass index. Factors like LV dimensions, LV ejection fraction, indexed
Table 2 Rest and stress echocardiographic data in the study sample $(\mathrm{n}=660)$

\begin{tabular}{|c|c|}
\hline Variable & Values \\
\hline LV ejection fraction & $62 \% \pm 1.5 \%$ \\
\hline Indexed LV-ESD $\left(\mathrm{cm} / \mathrm{m}^{2}\right)$ & $1.61 \pm 0.4$ \\
\hline Indexed LV-EDD $\left(\mathrm{cm} / \mathrm{m}^{2}\right)$ & $2.72 \pm 0.6$ \\
\hline Indexed left atrial area $\left(\mathrm{cm} / \mathrm{m}^{2}\right)$ & $13.6 \pm 3$ \\
\hline$E / e^{\prime}$ & $9.2 \pm 3$ \\
\hline Mitral ERO area $\left(\mathrm{cm}^{2}\right)$ & $0.45 \pm 0.2$ \\
\hline Mitral vena contracta $(\mathrm{cm})$ & $0.85 \pm 0.2$ \\
\hline Mitral leaflet flail & $187(28 \%)$ \\
\hline RVSP $(\mathrm{mmHg})$ & $31 \pm 12$ \\
\hline \multicolumn{2}{|l|}{ Tricuspid regurgitation } \\
\hline None-trivial & $415(63 \%)$ \\
\hline Mild & $152(23 \%)$ \\
\hline$\geq$ Moderate & $93(14 \%)$ \\
\hline \multicolumn{2}{|l|}{ Right ventricular dysfunction } \\
\hline None & $653(99 \%)$ \\
\hline Mild & $7(1 \%)$ \\
\hline LV-GLS & $-21.7 \% \pm 2 \%$ \\
\hline Maximum predicted heart rate & $96 \% \pm 22 \%$ \\
\hline$>85 \%$ maximum predicted heart rate & $653(99 \%)$ \\
\hline METs achieved & $9.9 \pm 3$ \\
\hline Heart rate recovery (beats/minute) & $34 \pm 14$ \\
\hline$\%$ age-gender predicted METs & $115 \% \pm 29 \%$ \\
\hline \multicolumn{2}{|l|}{ Age-gender predicted METs } \\
\hline$\leq 100 \%$ & $183(28 \%)$ \\
\hline$>100 \%$ & 477 (72\%) \\
\hline Stress-induced ischemia, \% & 0 \\
\hline \multicolumn{2}{|l|}{ Change in LV cavity size with stress } \\
\hline Decrease & $600(91 \%)$ \\
\hline Unchanged & $47(7 \%)$ \\
\hline Increased & $13(2 \%)$ \\
\hline Peak-stress RVSP (mmHg) & $46 \pm 15$ \\
\hline
\end{tabular}

LV, left ventricle; ESD, end-systolic dimension; EDD, enddiastolic dimension; ERO, effective regurgitant orifice; RVSP, right ventricular systolic pressure; METs, metabolic equivalents. 
Table 3 Univariable and multivariable logistic regression analysis for variables associated with not achieving $100 \%$ age-gender predicted metabolic equivalents

\begin{tabular}{|c|c|c|c|c|c|c|}
\hline \multirow{2}{*}{ Variable } & \multicolumn{3}{|c|}{ Univariable } & \multicolumn{3}{|c|}{ Multivariable } \\
\hline & Wald statistic & Odds ratio $(95 \% \mathrm{Cl})$ & $P$ value & Wald statistic & Odds ratio $(95 \% \mathrm{Cl})$ & $P$ value \\
\hline $\begin{array}{l}\text { Body mass index (for every } 1 \mathrm{~kg} / \mathrm{m}^{2} \\
\text { increase) }\end{array}$ & 22.7 & $1.12(1.07-1.17)$ & $<0.001$ & 17.6 & $1.11(1.06-1.17)$ & $<0.001$ \\
\hline Hypertension & 0.13 & $1.07(0.75-1.51)$ & 0.71 & - & - & - \\
\hline Smoking history & 0.17 & $0.88(0.46-1.77)$ & 0.62 & - & - & - \\
\hline Calcium channel blockers & 0.16 & $0.87(0.43-1.74)$ & 0.69 & - & - & - \\
\hline Higher indexed LV-ESD & 1.81 & $1.35(0.87-2.09)$ & 0.18 & - & - & - \\
\hline Higher indexed LA area & 2.1 & $1.02(0.99-1.04)$ & 0.15 & - & - & - \\
\hline Flail mitral leaflet & 2.0 & $1.31(0.90-1.91)$ & 0.16 & - & - & - \\
\hline Worsening resting LV-GLS & 47.6 & $1.43(1.29-1.59)$ & $<0.001$ & 39.6 & $1.40(1.21-1.55)$ & $<0.001$ \\
\hline$\geq$ Moderate TR & 0.19 & $1.01(0.62-1.67)$ & 0.67 & - & - & - \\
\hline $\begin{array}{l}\text { Resting RVSP (for every } 10 \text { mmHg } \\
\text { increase) }\end{array}$ & 10.3 & $1.27(1.10-1.52)$ & 0.001 & 5.7 & $1.22(1.02-1.49)$ & 0.02 \\
\hline $\begin{array}{l}\text { Post-stress RVSP (for every } 10 \\
\text { mmHg increase) }\end{array}$ & 7.8 & $1.18(1.04-1.42)$ & 0.01 & - & - & - \\
\hline
\end{tabular}

Due to inclusion of age and gender in the formula for calculation of \% predicted metabolic equivalents, they were not entered into the logistic regression analysis. Due to collinearity, only resting and not post-stress right ventricular systolic pressure was entered in the current multivariable analysis. LV, left ventricle; ESD, end systolic dimension; LA, left atrial; EF, ejection fraction; EROA, effective regurgitant orifice area; GLS, global longitudinal strain; TR, tricuspid regurgitation; RVSP, right ventricular systolic pressure.

LA area and mitral EROA were not significantly associated with exercise capacity in our study, likely because of a homogeneous study population with $\geq 3+$ primary $M R$, nondilated $L V$ and preserved $L V$ ejection fraction. Additionally, in order to avoid the potential confounding effect on resting LV-GLS measurements, we excluded patients with documented obstructive CAD, AF, left-sided valvular stenosis and bundle branch block.

Assessment of FC is important in asymptomatic patients with severe MR in particular, especially in the setting of a non-dilated $L V$ and preserved $L V$ ejection fraction, because of the ability of the heart to compensate for a long time before failing. In this setting, many patients may subtly reduce their exercise to prevent symptoms from occurring and thus as they are unaware of their symptoms, and unwittingly labeled as asymptomatic. Indeed, in a previous report, we have demonstrated that almost $1 / 3^{\text {rd }}$ of such patients, despite being deemed asymptomatic, failed to achieve $100 \%$ of their age-gender predicted METs (1). Previous smaller reports have also demonstrated that FC often tends to be abnormal in severe MR, even in asymptomatic patients with preserved EF $(4,8)$. In one study, where 134 asymptomatic severe MR patients with preserved EF were tested with cardiopulmonary exercise testing, FC showed a wide range, from above normal to the severely impaired range. Specifically, severely impaired capacity was found in $20-25 \%$ of these asymptomatic patients (4).

Several smaller studies have reported different and conflicting predictors of FC in MR patients $(4,8-15)$. Some reports have suggested that age, gender, and exercise cardiac output, early diastolic mitral annulus velocity, preoperative 
sinus rhythm, LV diastolic dysfunction, atrial fibrillation, forward stroke volume, exercise RVSP, low LA strain, brain natriuretic peptide predict $\mathrm{FC}$ in such patients, while $\mathrm{LV}$ ejection fraction, LA size, LV dimensions, mitral EROA, mitral regurgitant fraction, rest and exercise RVSP did not predict FC (4,8-15).

In previous smaller reports of asymptomatic severe MR patients with preserved $\mathrm{EF}$ who underwent exercise echocardiogram, LV-GLS was significantly associated with BNP levels, postoperative LV dysfunction, cardiac events (composite death, MV surgery or heart failure hospitalization) in follow up (23-25). In a recent study of 1,318 patients with $\geq 3+$ MR patients and preserved EF, we have demonstrated that addition of RVSP to STS significantly reclassified risk for longer-term mortality [integrated discrimination index 0.07 (0.04-0.09, $\mathrm{P}<0.001)]$. Furthermore, the risk of death significantly and continuously increased as RVSP increased from 35 to 60 $\mathrm{mmHg}$ with a $4 \%$ estimated hazard in the first year for RVSP of $50 \mathrm{mmHg}$ and $5 \%$ for RVSP of $60 \mathrm{mmHg}$ (26). In another recent study of 737 patients, we have demonstrated that a combination of resting LV-GLS and FC provide incremental prognostic value for the hard outcome of long-term death (17). In that study, we demonstrated that addition of \% age-gender predicted METs and resting LVGLS to STS, resting RVSP, LV end systolic dimension and mitral ERO increased the c-statistic for longerterm mortality from 0.61 to 0.69 and 0.78 (all $\mathrm{P}<0.01$ ). On quadratic spline analysis, risk of death progressively increased as resting LV-GLS worsened below $-21 \%$. Based on that finding, we hypothesized that LV-GLS could have a potential role in predicting $\mathrm{FC}$ in such patients, in addition to established predictors like RVSP. While it is easy to attribute impaired FC to obesity and deconditioning, there could be other potential factors at play. With the current study, we wanted to test the hypothesis if indeed there were general cardiac (and specific mitral-related) factors involved. Indeed, we demonstrate that LV-GLS was associated with exercise capacity, despite preserved LV systolic function, suggesting potential subclinical LV dysfunction prior to overt onset of LV systolic dysfunction.

To the best of our knowledge, the current study is the first to report an independent association between LVGLS and impaired FC in asymptomatic patients with severe primary MR, non-dilated LV and preserved EF. Indeed, the association between impaired FC and LV-GLS appears intuitive. In a prior histopathologic study, LV biopsies at the time of MV surgery in asymptomatic patients with chronic severe MR and normal $L V$ ejection fraction demonstrated more myofibrillar degeneration, higher xanthine oxidase and lipofuscin deposition indicating more oxidative stress compared to normal subjects (27). These histologic findings suggest that intrinsic LV dysfunction is already present in many patients with apparently normal LVEF and severe MR. Our study demonstrating that in this population of asymptomatic MR patients with preserved LVEF that global LV GLS is a major predictor of functional capacity supports the role of global LV GLS as a marker of impaired LV function with consequential impact on exercise capacity.

Currently, the definition of what constitutes "normal" LVGLS values remains controversial. The mean value of LVGLS in the current study $(-21.7 \%)$ was significantly higher than what was reported in a study of healthy individuals free of cardiovascular disease, where, similar to the current study, only VVI software was utilized (-17.3\%) (28). This is likely because LV-GLS appears to be loaddependent and hence the values in MR are higher. That previous study also demonstrated that LV-GLS values obtained using VVI software were similar for different echocardiography vendors (similar to our study) (28). However, a recent study has demonstrated that while there is a very strong correlation between LV-GLS values obtained using different strain analysis packages in same individuals, the absolute mean values vary between $-18 \%$ to $-21.5 \%$ (with a mean of $-20 \%$ for the Siemens strain software) (29). However, that study included patients with a wide spectrum of LV systolic function, rather than healthy individuals free of cardiovascular disease. The current data are hypothesis generating and require validation in the form of a prospective multicenter trial.

\section{Limitations}

This was an observational study from a single center with its inherent selection bias. We only included asymptomatic patients with $\geq 3+$ primary MR that subsequently underwent rest and post-exercise echocardiography. Because we only included patients with $\geq 3+$ primary MR, the study population was homogeneous with regards to the degree of MR and hence, mitral EROA was likely not a significant predictor of exercise capacity. At our center, during the time frame of the current study, approximately 8,500 patients were evaluated for significant primary MR, of which only $\sim 10 \%$ underwent treadmill echocardiography in situations where there was ambiguity in terms of symptoms and more information was needed to aid in timing of MV surgery, 
as reported previously (1). Therefore, patients who have been more symptomatic at baseline did not have the test and are not included in our study. However, this has inherently helped us to focus on a population in whom uncertainties exist about optimal management strategies. We included patients over a broad time-frame and not all the data (biomarker, volumetric LV/LA data and right ventricular measurements) were performed in all patients. The frame rate of stored data in DICOM format was above 30 frames/second which is low for good analysis. LV-GLS is load-dependent and could differ with alterations in blood pressure. However, simultaneous blood pressures were not routinely measured. In addition, we do not report results of LV-GLS at peak-stress. However, it can be argued that LV-GLS measurement at peak-stress is difficult and would result in many more excluded patients (due to non-tracked segments). Also, at peak stress, the window of opportunity to acquire clinical data (wall motion analysis and RVSP) is very short before heart rate drops significantly. As a result, adding LV-GLS measurements could potentially impact the quality of data acquisition. Also, exercise capacity reflects the overall function of cardiovascular, respiratory and musculoskeletal systems, and other possible etiologies of impaired exercise capacity could have played an important role.

\section{Conclusions}

In patients with severe primary $M R$, non-dilated $L V$ and preserved LV ejection fraction undergoing restexercise echocardiography, worsening resting LV-GLS is significantly associated with reduced $\mathrm{FC}$, independent and incremental to other known predictors. These findings potentially support the use of $\mathrm{LV}$ global longitudinal strain in detecting subtle myocardial dysfunction that could have a tangible functional impact.

\section{Acknowledgements}

The study was supported, in part, by a philanthropic gift from Reginald and Jamie Baxter family to Dr. Desai. Dr. Desai is supported in part by the Haslam Family endowed chair in cardiovascular medicine.

\section{Footnote}

Conflicts of Interest: MA Gillinov reports following financial conflicts of interest-speakers' bureau for Atricure,
Edwards, Medtronic and St. Jude's Medical. He also reports equity stake in Pleuraflow. The other authors have no conflicts of interest to declare.

Ethical Statement: The study was approved by the institutional review board with waiver of individual informed consent.

\section{References}

1. Naji P, Griffin BP, Asfahan F, et al. Predictors of long-term outcomes in patients with significant myxomatous mitral regurgitation undergoing exercise echocardiography. Circulation 2014;129:1310-9.

2. Naji P, Griffin BP, Barr T, et al. Importance of exercise capacity in predicting outcomes and determining optimal timing of surgery in significant primary mitral regurgitation. J Am Heart Assoc 2014;3:e001010.

3. Naji P, Asfahan F, Barr T, et al. Impact of duration of mitral regurgitation on outcomes in asymptomatic patients with myxomatous mitral valve undergoing exercise stress echocardiography. J Am Heart Assoc 2015;4(2). pii: e001348.

4. Messika-Zeitoun D, Johnson BD, Nkomo V, et al. Cardiopulmonary exercise testing determination of functional capacity in mitral regurgitation: physiologic and outcome implications. J Am Coll Cardiol 2006;47:2521-7.

5. Magne J, Lancellotti P, Pierard LA. Exercise pulmonary hypertension in asymptomatic degenerative mitral regurgitation. Circulation 2010;122:33-41.

6. Magne J, Lancellotti P, Pierard LA. Exercise-induced changes in degenerative mitral regurgitation. J Am Coll Cardiol 2010;56:300-9.

7. Nishimura RA, Otto CM, Bonow RO, et al. 2014 AHA/ ACC Guideline for the Management of Patients With Valvular Heart Disease: A Report of the American College of Cardiology/American Heart Association Task Force on Practice Guidelines. Circulation 2014;129:e521-643.

8. Leung DY, Griffin BP, Snader CE, et al. Determinants of functional capacity in chronic mitral regurgitation unassociated with coronary artery disease or left ventricular dysfunction. Am J Cardiol 1997;79:914-20.

9. Kim HK, Kim YJ, Chung JW, et al. Impact of left ventricular diastolic function on exercise capacity in patients with chronic mitral regurgitation: an exercise echocardiography study. Clin Cardiol 2004;27:624-8.

10. Haluska BA, Short L, Marwick TH. Relationship of ventricular longitudinal function to contractile reserve in patients with mitral regurgitation. Am Heart J 2003;146:183-8. 
11. Yusoff R, Clayton N, Keevil B, et al. Utility of plasma $\mathrm{N}$-terminal brain natriuretic peptide as a marker of functional capacity in patients with chronic severe mitral regurgitation. Am J Cardiol 2006;97:1498-501.

12. Suzuki K, Izumo M, Yoneyama K, et al. Influence of exercise-induced pulmonary hypertension on exercise capacity in asymptomatic degenerative mitral regurgitation. J Cardiol 2015;66:246-52.

13. Lee SP, Kim YJ, Lee JM, et al. Association of heart rhythm with exercise capacity after operation for chronic mitral regurgitation. Ann Thorac Surg 2012;93:1888-95.

14. Vaturi M, Hadar T, Yedidya I, et al. The association of left atrial volume with exercise capacity in patients with chronic severe mitral regurgitation. Isr Med Assoc J 2010;12:150-3.

15. Yang LT, Shih JY, Liu YW, et al. Effects of left atrial strain on functional capacity in chronic severe mitral regurgitation. Int J Cardiol 2013;168:e151-3.

16. Marwick TH, Leano RL, Brown J, et al. Myocardial strain measurement with 2-dimensional speckle-tracking echocardiography: definition of normal range. JACC Cardiovasc Imaging 2009;2:80-4.

17. Mentias A, Naji P, Gillinov AM, et al. Strain Echocardiography and Functional Capacity in Asymptomatic Primary Mitral Regurgitation With Preserved Ejection Fraction. J Am Coll Cardiol 2016;68:1974-86.

18. Lang RM, Badano LP, Mor-Avi V, et al. Recommendations for cardiac chamber quantification by echocardiography in adults: an update from the American Society of Echocardiography and the European Association of Cardiovascular Imaging. J Am Soc Echocardiogr 2015;28:1-39.e14.

19. Zoghbi WA, Enriquez-Sarano M, Foster E, et al. Recommendations for evaluation of the severity of native valvular regurgitation with two-dimensional and Doppler echocardiography. J Am Soc Echocardiogr 2003;16:777-802.

20. Rudski LG, Lai WW, Afilalo J, et al. Guidelines for the echocardiographic assessment of the right heart in adults: a report from the American Society of Echocardiography endorsed by the European Association of Echocardiography, a registered branch of the European Society of Cardiology, and the Canadian Society of Echocardiography. J Am Soc Echocardiogr 2010;23:685-713; quiz 686-8.

21. Pellikka PA, Nagueh SF, Elhendy AA, et al. American Society of Echocardiography recommendations for performance, interpretation, and application of stress echocardiography. J Am Soc Echocardiogr 2007;20:1021-41.
22. Kusunose K, Goodman A, Parikh R, et al. Incremental prognostic value of left ventricular global longitudinal strain in patients with aortic stenosis and preserved ejection fraction. Circ Cardiovasc Imaging 2014;7:938-45.

23. Lancellotti P, Cosyns B, Zacharakis D, et al. Importance of left ventricular longitudinal function and functional reserve in patients with degenerative mitral regurgitation: assessment by two-dimensional speckle tracking. J Am Soc Echocardiogr 2008;21:1331-6.

24. Donal E, Mascle S, Brunet A, et al. Prediction of left ventricular ejection fraction 6 months after surgical correction of organic mitral regurgitation: the value of exercise echocardiography and deformation imaging. Eur Heart J Cardiovasc Imaging 2012;13:922-30.

25. Magne J, Mahjoub H, Dulgheru R, et al. Left ventricular contractile reserve in asymptomatic primary mitral regurgitation. Eur Heart J 2014;35:1608-16.

26. Mentias A, Patel K, Patel H, et al. Effect of Pulmonary Vascular Pressures on Long-Term Outcome in Patients With Primary Mitral Regurgitation. J Am Coll Cardiol 2016;67:2952-61.

27. Ahmed MI, Gladden JD, Litovsky SH, et al. Increased oxidative stress and cardiomyocyte myofibrillar degeneration in patients with chronic isolated mitral regurgitation and ejection fraction >60\%. J Am Coll Cardiol 2010;55:671-9.

28. Fine NM, Shah AA, Han IY, et al. Left and right ventricular strain and strain rate measurement in normal adults using velocity vector imaging: an assessment of reference values and intersystem agreement. Int J Cardiovasc Imaging 2013;29:571-80.

29. Farsalinos KE, Daraban AM, Unlu S, et al. Head-to-Head Comparison of Global Longitudinal Strain Measurements among Nine Different Vendors: The EACVI/ASE InterVendor Comparison Study. J Am Soc Echocardiogr 2015;28:1171-81.e2.

Cite this article as: Mentias A, Alashi A, Naji P, Gillinov AM, Rodriguez LL, Mihaljevic T, Suri RM, Grimm RA, Svensson LG, Griffin BP, Desai MY. Exercise capacity in asymptomatic patients with significant primary mitral regurgitation: independent effect of global longitudinal left ventricular strain. Cardiovasc Diagn Ther 2018;8(4):460-468. doi: 10.21037/ cdt.2018.05.03 